\title{
LA GIUSTIZIA ELETTORALE IN ITALIA E IN MESSICO: UN PRIMO CONFRONTO
}

ELECTORAL JUSTICE IN ITALY AND MEXICO: A FIRST COMPARISON

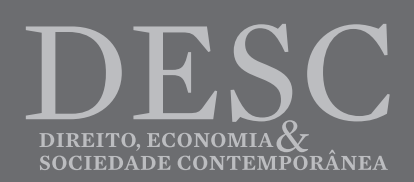




\title{
LA GIUSTIZIA ELETTORALE IN ITALIA E IN MESSICO: UN PRIMO CONFRONTO \\ ELECTORAL JUSTICE IN ITALY AND MEXICO: A FIRST COMPARISON
}

\section{GIORGIO SOBRINO}

Università degli Studi di Torino giorgio.sobrino@unito.it https://www.dg.unito.it/do/docenti.pl/Show?_id=gsobrino

\begin{abstract}
Riassunto: L'articolo propone un confronto generale tra il sistema di giustizia elettorale attualmente vigente in Italia e quello del Messico. Muovendo da un inquadramento preliminare della nozione di giustizia elettorale, lo scritto presenta una ricostruzione complessiva degli istituti e dei meccanismi di tutela dei diritti politico- elettorali dei cittadini, e degli organi ad essi preposti, previsti da questi due ordinamenti, ed esprime, in conclusione, alcune considerazioni finali in un'ottica appunto di confronto tra essi. Parole- chiave: giustizia elettorale, diritto di voto, diritti politico- elettorali
\end{abstract}

\begin{abstract}
The article proposes a general comparison between the electoral justice systems currently in force in Italy and in Mexico. Starting from a preliminary framework of the notion of electoral justice, the paper presents an overall reconstruction of the institutions and the mechanisms for the protection of the political-electoral rights of citizens, and of the bodies responsible for them, provided by these two legal systems, and expresses, in conclusion, some final considerations with a view to comparing them.
\end{abstract}

Keywords: electoral justice, right to vote, political-electoral rights

\section{1. - Introduzione}

Scopo di questo scritto - che rappresenta il frutto di un periodo di ricerca che ho avuto la fortuna di svolgere presso l'Instituto de Investigaciones Juridicas dell'U.N.A.M. all'inizio di quest'anno ${ }^{1}$ - è operare un primo confronto tra il sistema italiano e quello messicano di giustizia elettorale.

1 Mi sia consentito ringraziare qui i professori Pedro Salazar Ugarte (Direttore dell'Instituto de Investigaciones Juridicas) e Lorenzo Cordova Vianello (Presidente dell'Instituto Nacional Electoral del Messico) per la cortese ospitalità, ed il prof. Michelangelo Bovero per avere reso possibile questa ricerca. 
Si tratta di un primo confronto (ritengo opportuno precisarlo) per due motivi. Da un lato, per i limiti di spazio di questo articolo, che impongono di fermarsi agli aspetti generali - ma essenziali - dell'argomento. Ma soprattutto (e dall'altro lato) perché la giustizia elettorale, della quale i sistemi qui considerati sono due esempi concreti, è un tema (o meglio, un problema) vasto e complesso, che richiede analisi e riflessioni di respiro più ampio. Esse coinvolgono infatti, tra gli altri profili, la concezione dei diritti politici e del loro "peso" rispetto alle altre categorie di diritti costituzionali nei diversi contesti ${ }^{2}$, nonché l'assetto delle relazioni tra i poteri (pubblici e non solo) nei diversi Stati. Nel nostro caso, in Italia ed in Messico.

Poiché la mia ricerca su questi profili è tuttora in corso dopo il periodo di analisi "dall'interno" dell'ordinamento messicano, in questa sede mi limiterò - muovendo da un inquadramento generale della nozione di giustizia elettorale - a presentare una panoramica degli istituti e meccanismi di tutela dei diritti politico- elettorali previsti da tale ordinamento e da quello italiano, per poi esprimere qualche considerazione finale in un'ottica, appunto, di confronto tra essi.

\section{2. - La nozione di giustizia elettorale; l'oggetto della comparazione}

Secondo la dottrina latinoamericana, ed in particolare messicana, che si è occupata specificamente di questo tema ${ }^{3}$, «giustizia elettorale» è un'espressione (ed un concetto, o "categoria") non univoca. Ad essa, infatti, vengono attribuiti significati almeno in parte differenti.

In una prima accezione - che potremmo definire «stretta» -, la giustizia elettorale consiste nei diversi meccanismi di natura processuale volti a garantire che gli atti ed i procedimenti elettorali si conformino alla Costituzione ed alla legge, nonché a proteggere e, se necessario, a «ripristinare» l'effettivo esercizio dei diritti politico- elettorali dei cittadini. Intesa in questo modo, essa comprende tutti gli strumenti «giuridico- processuali» di controllo di costituzionalità e di legittimità in materia elettorale (azioni, ricorsi, reclami, ecc.) e, correlativamente, di difesa dei diritti politico- elettorali individuali ${ }^{4}$.

In una seconda accezione (solo in parte coincidente con la prima), la giustizia elettorale è l'insieme dei «mezzi d'impugnazione» in ambito elettorale, di natura non soltanto giurisdizionale, ma anche amministrativa o «legislativa» (cioè le impugnazioni davanti agli organi del potere legislativo, come avviene per esempio in Italia per gli atti riguardanti le elezioni del Parlamento ${ }^{5}$. In questa prospettiva, essa coincide con la nozione di contenzioso elettorale

2 Soprattutto dal punto di vista del loro rispettivo grado di effettività (posto che ha poco senso "affermare" un diritto in un documento costituzionale, senza al contempo predisporre degli adeguati strumenti di tutela in concreto del diritto stesso).

3 J. Orozco Henriquez, Justicia electoral comparada de América Latina, Mexico, U.N.A.M., Instituto de Investigaciones Juridicas, 2019, 9 (che costituisce il principale riferimento di questo inquadramento preliminare, nonché della trattazione del sistema messicano che verrà svolta in seguito).

4 Ibidem, in particolare 9 e 11.

5 V. infra, $\S \S 3-3.1$. 
(appunto, giurisdizionale o di altro genere), volto a confermare, modificare o annullare/revocare gli atti del procedimento elettorale oggetto di impugnativa ${ }^{6}$.

In una terza accezione - che potremmo invece definire «ampia»-, «giustizia elettorale» è invece l'insieme delle misure di varia natura (non soltanto quindi processuali o, comunque, inerenti al contenzioso elettorale), finalizzate alla «massima realizzazione della democrazia rappresentativa o degli istituti di democrazia semidiretta che utilizzano la tecnica del suffragio» ${ }^{7}$ , come ad esempio il referendum. Intesa in questo significato, ne fanno parte anche la legge elettorale (che dovrebbe prevedere un sistema elettorale «adeguato» allo scopo indicato), le norme sul diritto di associazione e le altre libertà politiche, la legge sul finanziamento dei partiti e delle campagne elettorali (dei partiti stessi e dei singoli candidati).

Tra questi diversi significati, nel presente lavoro utilizzerò il secondo sopra indicato giustizia elettorale come insieme dei «mezzi d'impugnazione» in materia elettorale -, perché comprensivo dei rimedi non giurisdizionali ed in grado, dunque, di abbracciare il sistema italiano (o meglio, la sua parte più importante: le regole sull'impugnazione degli atti delle elezioni del Parlamento nazionale), oltre a quello messicano. Nei paragrafi seguenti, perciò, cercherò di presentare - e di mettere a confronto - i mezzi d'impugnazione previsti da questi due ordinamenti (di qualsiasi natura essi siano) per le diverse consultazioni elettorali (nazionali, locali, ecc.).

In particolare, metterò in evidenza gli organi davanti ai quali le impugnazioni devono essere proposte, e deputati quindi a decidere su di esse. Uno dei criteri principali di classificazione dei sistemi di giustizia elettorale a livello comparato utilizzati dalla dottrina, infatti, consiste nella natura dell'organo «incaricato di emettere la decisione finale sulle impugnazioni relative alla validità ed ai risultati delle elezioni $»^{8}$. Al riguardo, si distingue tra sistemi che affidano tale decisione agli organi legislativi (assemblee parlamentari, essenzialmente); ad un organo giurisdizionale (distinguendosi - a sua volta - a seconda del tipo di organo giurisdizionale competente: giudice ordinario; giudice amministrativo; tribunale «specializzato» in materia elettorale; tribunale costituzionale); ad un organo amministrativo (eventualmente dotato di compiti giurisdizionali, o «para- giurisdizionali»); ad un organo «transitorio ad hoc» ${ }^{9}$. Ne derivano modelli diversi di sistemi di giustizia elettorale, ai quali anche l'ordinamento italiano e quello messicano possono essere ascritti.

Per quanto riguarda, poi, gli atti suscettibili di impugnazione (tramite gli strumenti e gli

6 J. Orozco Henriquez, Justicia electoral comparada, cit., 13.

7 Ibidem, $14 \mathrm{~s}$.

8 Ibidem, 54.

9 Ibidem, 55. In una prospettiva analitica simile, un altro costituzionalista messicano, Héctor Fix- Zamudio, distingue tra contenzioso elettorale «politico», «giuridico» (davanti ad organi amministrativi o giurisdizionali) e «misto»: H. Fix- Zamudio, Introduccion a la teoria de los recursos en el contencioso electoral, 5 ss. 
istituti del contenzioso elettorale di cui sopra), mi concentrerò su quelli relativi ai risultati delle elezioni - dunque sul contenzioso elettorale inteso nel suo significato più stretto - , ma anche alla «preparazione» di esse (provvedimenti sull'ammissione delle liste, delle candidature, ecc.). La dottrina latinoamericana, peraltro (soprattutto quella che accoglie l'accezione più ampia di giustizia elettorale sopra riportata), considera parte del sistema di giustizia elettorale anche gli strumenti di tutela riconosciuti ai cittadini nei confronti degli atti diversi da quelli del procedimento elettorale vero e proprio, e compresi - dal punto di vista temporale - tra un'elezione e l'altra. In questa prospettiva, vengono da essa esaminati, per esempio, i rimedi contro i provvedimenti in materia di registrazione dei partiti e delle associazioni politiche (in Messico adottati, come vedremo, dall'Instituto Nacional Electoral - I.N.E.), di controllo nei confronti delle loro attività e di irrogazione delle eventuali sanzioni (sempre da parte dell'I.N.E.), nonché sull'utilizzo delle risorse pubbliche per il finanziamento dell'attività politica "ordinaria", svolta anche da singoli cittadini ${ }^{10}$. Un'indagine di questo tipo, sebbene interessante, sarebbe troppo ampia per il presente lavoro; e d'altra parte, rischierebbe di far perdere di vista quello che è il "nucleo" (il "proprium") della giustizia elettorale, cioè - come si è visto sopra - i meccanismi di tutela connessi alle elezioni in senso stretto e, correlativamente, di garanzia dei diritti politicoelettorali dei consociati ${ }^{11}$.

\section{3. - Il sistema italiano di giustizia elettorale: caratteri generali e linee evolutive} recenti

Il sistema italiano di giustizia elettorale si connota tradizionalmente - tra i diversi “modelli" soprarichiamati-come un esempio di giustizia elettorale $\langle p o l i t i c a\rangle{ }^{12}$. Le impugnazioni riguardanti l'elezione dei membri del Parlamento (formato dalla Camera dei deputati e dal Senato della Repubblica), le più importanti in un contesto di forma di governo parlamentare, sono infatti devolute, per espressa disposizione costituzionale, alle stesse Camere; la relativa decisione è insindacabile dagli altri organi e poteri dello Stato (art. 66 Cost.: «Ciascuna Camera giudica dei titoli di ammissione dei suoi componenti e delle cause sopraggiunte di ineleggibilità e di incompatibilità»).

Questa scelta del Costituente - che trova riscontro in altri ordinamenti, anche

10 J. Orozco Henriquez, Justicia electoral comparada, cit., 23 e, più specificamente, 230 ss.

11 Sulla distinzione (sempre sul piano teorico- generale) tra diritti «politici» e diritti «politico- elettorali» dei cittadini - categoria, quest'ultima, più ristretta e legata direttamente allo svolgimento delle consultazioni elettorali - v. ancora J. Orozco Henriquez, Justicia electoral comparada, cit., 20.

12 Ibidem, 55 e 59. Da una prospettiva interna, propria invece della dottrina costituzionalistica italiana, v. (tra i lavori indicati nella bibliografia in calce a questo articolo) L. Elia, Elezioni politiche (contenzioso), in Enciclopedia del diritto, Milano, Giuffrè, 1965 , vol. XIV, 748 ss.; L. Imarisio, Ineleggibilità e incompatibilità politico- istituzionali, Napoli, Jovene, 2008, 295 ss.; M. Cerase, Sviluppi e contrasti in tema di verifica dei poteri, in Diritto pubblico, 2004, 662 ss. 
latinoamericani ${ }^{13}$ - è il frutto di un'eredità storica dello Stato liberale ${ }^{14}$. Essa, invero, risponde ad una logica (tipica, appunto, della concezione liberale dello Stato) di «rigida» separazione dei poteri: gli organi ed i poteri diversi, in particolare quelli giudiziari, non possono ingerirsi con i loro provvedimenti nella sfera della politica, caratterizzata da piena autonomia.

Al di là della previsione appena richiamata, la Costituzione italiana - a differenza delle Carte dei Paesi dell'America latina e, per quanto qui interessa, di quella messicana - non contiene inoltre norme sul contenzioso elettorale e, dunque, sulla tutela dei diritti politici connessi allo svolgimento delle elezioni (o «politico- elettorali»). Essa si limita a riconoscere il diritto di elettorato attivo e passivo dei cittadini (differenziandolo, solo per i due rami del Parlamento, in ragione dell'età: artt. 56 e 58) e a dettare alcuni principi relativi al voto in generale: «il voto è personale ed eguale, libero e segreto» (art. 48). Da questi principi la giurisprudenza della Corte Costituzionale, in particolare negli ultimi anni, ha ricavato alcune implicazioni rilevanti per la tutela in concreto del diritto di voto, con riguardo essenzialmente alle caratteristiche costituzionalmente necessarie del sistema elettorale per l'elezione del Parlamento ${ }^{15}$. Il quadro costituzionale è comunque, in complesso, scarno.

Occorre tuttavia evidenziare che nel corso del tempo il sistema italiano di giustizia elettorale si è evoluto in modo significativo (sebbene - come si dirà in conclusione - non "risolutivo" ai fini della sua classificazione generale, che resta quella indicata sopra).

Da un lato, infatti, la legislazione ordinaria ha previsto, già da molti anni, un insieme articolato di mezzi d'impugnazione davanti agli organi giurisdizionali (ordinari ed amministrativi) degli atti relativi alle elezioni a livello territoriale (regionali, provinciali e comunali: cc.dd. elezioni «amministrative»), nonché dei membri del Parlamento europeo spettanti all'Italia: sicché si può affermare che l'attuale "modello" italiano di giustizia elettorale è - almeno in parte - «giurisdizionalizzato» (oppure, in termini forse più rigorosi considerata la centralità delle elezioni del Parlamento nazionale, non integralmente «politico»).

Dall'altro lato, per effetto di due recenti (ed assai importanti anche al di là del tema della giustizia elettorale ${ }^{16}$ ) pronunce della Corte Costituzionale - le già ricordate sentenze n. $1 / 2014$ e $35 / 2017$ - è stata riconosciuta ai cittadini la possibilità di far valere davanti al

13 In particolare nell'ordinamento argentino: la Camera dei deputati ed il Senato giudicano dell'elezione dei loro rispettivi membri, ed il Parlamento in seduta comune effettua il conteggio definitivo dei voti e la proclamazione del Presidente (cfr. J. Orozco Henriquez, Justicia electoral comparada, cit., 59 s.).

14 Essa risale, precisamente, al 1868, quando venne introdotto in Italia l'istituto della «verifica dei poteri», su ispirazione del sistema inglese: L. Imarisio, Ineleggibilità e incompatibilità, cit., 295.

15 Sentenze n. 1/2014 e 35/2017, su cui ci si soffermerà in seguito.

16 In quanto tali decisioni investono, e possono cambiare profondamente, l'intero sistema di giustizia costituzionale italiano: v., al riguardo (tra i lavori richiamati nella bibliografia in calce), R. Bin, La Corte Costituzionale può introdurre con una sentenza il ricorso diretto di costituzionalità delle leggi?, in laCostituzione.info, 13 gennaio 2017, nonché G. Sobrino, Il problema dell'ammissibilità delle questioni di legittimità costituzionale della legge elettorale alla luce delle sentenze n. 1/2014 e n. 35/2017 e le sue possibili ricadute: dalla (non più tollerabile) "zona franca" alla (auspicabile) "zona a statuto speciale” della giustizia costituzionale?, in federalismi. it, n. 15/2017. 
giudice ordinario la «lesione del diritto politico di voto» anche in relazione alle elezioni del Parlamento. In particolare, ciascun «cittadino elettore» ha ora il diritto di agire in sede giudiziaria per far accertare l'eventuale violazione del proprio diritto di «votare conformemente alla Costituzione», cioè «in modo libero e diretto», «personale ed eguale» (secondo il dettato dell'art. 48 Cost.); e per attivare, in questo modo, un giudizio (c.d. «incidentale») di legittimità costituzionale della legge elettorale davanti alla stessa Corte Costituzionale, che potrà dichiarare tale legge illegittima. Si tratta di un'innovazione che (almeno fino a questo momento, stando alla motivazione delle due sentenze citate) non è idonea ad incidere sul risultato delle elezioni: ossia sulla c.d. «proclamazione» dei deputati e dei senatori eletti. La competenza esclusiva delle Camere in ordine alla «verifica dei poteri» (cioè alla verifica della regolarità delle procedure elettorali e della sussistenza effettiva «dei titoli di ammissione» dei loro membri, di cui al già citato art. 66 Cost.), nonché a decidere contro le eventuali impugnazioni in merito, non è messa in discussione. Tuttavia, nella misura in cui essa consente ai cittadini, sostanzialmente, di impugnare la legge elettorale e di farla eliminare dall'ordinamento ove in contrasto con i principi costituzionali sul diritto di voto, rappresenta un importante strumento di tutela di tale diritto, appunto in relazione (anche) alle elezioni del Parlamento.

Alla luce di quanto precede, pare opportuno, sul piano sistematico, trattare separatamente gli strumenti di tutela riconosciuti agli elettori in Italia nei confronti degli atti delle elezioni locali (o «amministrative») e dei membri del Parlamento europeo (§ 3.1) e, dall'altro lato, quelli riguardanti l'elezione del Parlamento nazionale (§ 3.2); per poi passare all'esame del sistema di giustizia elettorale vigente in Messico (§ 4).

\section{1. - Gli strumenti di tutela dei diritti politico- elettorali nelle elezioni locali e del}

\section{Parlamento europeo}

Iniziando dalla tutela dei diritti politico- elettorali (diritto di voto, in primo luogo; ma anche di elettorato passivo ed altri) nelle elezioni diverse da quelle del Parlamento nazionale ${ }^{17}$, essa - come già accennato - nell'attuale ordinamento italiano è affidata interamente agli organi giurisdizionali. Come in altre materie, vi è al riguardo, peraltro, un «riparto di competenze» tra il giudice ordinario ed il giudice («speciale») amministrativo: davanti ad essi devono infatti essere impugnati atti elettorali diversi, ed azionati diritti (o altre situazioni giuridiche soggettive) diversi.

Nello specifico, davanti al giudice ordinario devono essere proposti gli eventuali ricorsi aventi ad oggetto l'eleggibilità dei candidati alle cariche elettive locali (art. 82 d.P.R.

17 V., in particolare (tra i lavori richiamati nella bibliografia in calce), E. Lehner, Le garanzie dei diritti elettorali, Roma, Aracne, 2012, 161 ss.; L. Imarisio, Ineleggibilità e incompatibilità, cit., 303 ss.; E. Catelani - E. Donati - M.C. Grisolia (cur.), La giustizia elettorale, Napoli, Editoriale Scientifica, 2013. 
n. 570/1960 per i Comuni; art. 19 legge n. 108/1968 per le Regioni ${ }^{18}$ ) o al Parlamento europeo (art. 44 legge n. 18/1979). Tramite questi ricorsi i «cittadini elettori» dell'Ente territoriale in questione - ma anche ogni altra persona «vi abbia diretto interesse» (art. 82 d.P.R. n. 570/1960, cit.) - possono contestare l'esistenza in capo ai candidati dichiarati eletti di una (o più) delle cause di «incandidabilità», «ineleggibilità» $\mathrm{O}$ «incompatibilità» previste dalla legge. Ciò avviene impugnando la delibera adottata in materia dal Consiglio comunale o dal Consiglio regionale, che all'atto del loro insediamento esaminano $\mathrm{i}$ «titoli di ammissione» dei loro membri (come le Camere del Parlamento ai sensi dell'art. 66 Cost., sopra citato). Se il giudice accoglie il ricorso, il candidato viene dichiarato ineleggibile e, perciò, «sostituito» con quello che avrebbe dovuto essere proclamato eletto al suo posto (art. 22 d.lgs. n. 150/2011). Per quanto riguarda i membri del Parlamento europeo, invece, non è necessario impugnare alcun atto; i ricorsi devono essere proposti entro trenta giorni dalla pubblicazione dei nomi degli eletti (art. 23 dello stesso d.lgs. n. 150/2011).

Occorre ricordare, inoltre, che l'azione giudiziaria volta a far valere l'esistenza di una causa di «ineleggibilità» (o di «incompatibilità») dell'organo elettivo locale può essere esercitata dal cittadino anche in un momento successivo all'inizio del relativo mandato, a fronte di situazioni illegittime sopravvenute. Infatti, anche in questo caso la mancata eliminazione della causa di «ineleggibilità» (o di «incompatibilità») da parte dell'organo coinvolto ne comporta la «decadenza» (art. 68 d.lgs. n. 267/2000 per i Comuni; art. 7 legge n. 154/1981 per le Regioni). E' previsto, in proposito, un procedimento di contestazione della condizione di ineleggibilità/ incompatibilità all'interno del Consiglio comunale o regionale, al cui esito viene adottata una delibera che può essere impugnata davanti al giudice ordinario entro trenta giorni (art. 22 d.lgs. n. 150/2011).

Il giudice amministrativo, invece, è competente a decidere i ricorsi concernenti tutte le «operazioni elettorali» successive alla convocazione dei «comizi» - sino all'atto di «proclamazione» degli eletti - per le elezioni locali e del Parlamento europeo (artt. 126 del codice del processo amministrativo e 83 d.P.R. n. 570/1960). Più precisamente, dal 2010 è riconosciuta agli aspiranti candidati la possibilità di impugnare immediatamente ${ }^{19}$ (prima, quindi, dello svolgimento della consultazione elettorale) i provvedimenti di esclusione delle liste o dei candidati adottati dall'Ufficio elettorale. Il relativo giudizio è assai rapido: l'udienza si tiene entro tre giorni dal deposito del ricorso, e la sentenza è pubblicata subito dopo e comunicata all'Ufficio elettorale (art. 129 c.p.a.). Già da prima del 2010, invece, è prevista l'impugnabilità - da parte dei candidati e degli elettori dell'Ente territoriale coinvolto, o di tutti i cittadini

18 Per le Province, invece, la legge n. 56/2014 ha abolito l'elettività diretta degli organi, quindi il problema dell'eventuale ineleggibilità assume contorni diversi.

19 Per l'esattezza, entro tre giorni dalla loro adozione (art. 129 c.p.a.). 
nel caso delle elezioni del Parlamento U.E. - di tutti gli altri atti del procedimento elettorale, compresa la «proclamazione» degli eletti, nel termine di trenta giorni da quest'ultima. Anche in questo caso, la procedura davanti al Tribunale Amministrativo Regionale competente (sempre il T.A.R. del Lazio per le elezioni europee) è "accelerata" rispetto ai giudizi comuni, in chiave evidentemente - di garanzia della certezza dei risultati elettorali e, dall'altro lato, dell'effettività della tutela del diritto di voto degli elettori (art. 130 c.p.a.).

Infine, va ricordato che tanto il giudice ordinario quanto il giudice amministrativo, all'interno dei rispettivi giudizi, possono investire la Corte Costituzionale di eventuali questioni di costituzionalità della disciplina elettorale che essi sono chiamati ad applicare. Se la questione è effettivamente «rilevante» per la decisione sul ricorso del cittadino, la Corte la esamina ${ }^{20}$, "entrando" quindi, a sua volta, nel "campo" delle elezioni locali e del Parlamento U.E.; e potendo dichiarare eventualmente l'illegittimità costituzionale delle norme del sistema elettorale o di altre, concorrendo così a tutelare i diritti politici del cittadino sanciti dalla Costituzione.

\section{2. - La «verifica dei poteri» e la tutela del diritto di «votare conformemente alla}

\section{Costituzione» nelle elezioni politiche nazionali}

Passando agli istituti di garanzia operanti in Italia, allo stato attuale, in riferimento all'elezione del Parlamento nazionale, essi - come rilevato sopra - sono fondamentalmente due: uno tradizionale, e previsto espressamente dalla Costituzione - la «verifica dei poteri» (art. 66 , cit.) -; l'altro invece recente, e introdotto in via "pretoria" dal circuito giudici comuni- Corte Costituzionale - l'azione di accertamento della «pienezza» del diritto di voto, ossia del diritto dell'elettore di «votare conformemente alla Costituzione» (sentenze n. 1/2014 e 35/2017 della Corte Costituzionale) -.

Nello specifico, la «verifica dei poteri», in primo luogo, consiste in un procedimento interno alle due Camere volto, da una parte, a valutare $\mathrm{i}$ «titoli di ammissione» degli eletti (di cui parla l'art. 66 Cost.) e l'esistenza di eventuali situazioni di «ineleggibilità» e «incompatibilità»; dall'altra parte (e sostanzialmente), a controllare la regolarità del procedimento elettorale e la correttezza dei risultati. In concreto, la procedura prevede una fase di «delibazione» dell'elezione di ogni candidato - e di eventuale contestazione di essa da parte di chi vi abbia interesse davanti alle Giunte della Camera e del Senato competenti in materia, ed una successiva fase di deliberazione definitiva da parte delle due Assemblee (che possono "ribaltare" la decisione della Giunta, anche in caso di convalida dell'elezione). Le Giunte sono composte da parlamentari nominati dai Presidenti delle rispettive Camere: in ragione di ciò esse tendono ad operare sulla base di criteri e logiche politiche (soprattutto quando le loro decisioni sono suscettibili di

20 Giudizio «incidentale» di legittimità costituzionale, tipico dell'ordinamento italiano. 
incidere sugli equilibri all'interno dell' Assemblea ${ }^{21}$ ), nonostante i relativi procedimenti abbiano via via assunto, per effetto di successive riforme dei regolamenti parlamentari, un contenuto e garanzie sostanzialmente "giurisdizionali" (è proprio il caso, in particolare, del «giudizio di contestazione») $)^{22}$.

Come già ricordato, inoltre, la decisione assunta dall'Aula parlamentare sulla convalida o meno dell'elezione è insindacabile dagli altri organi e poteri dello Stato (compresa, fino a questo momento, la Corte Costituzionale $\left.{ }^{23}\right)$ : il che conferma la natura «politica» di questo istituto e, conseguentemente, del "modello" italiano di giustizia elettorale.

Per quanto riguarda, in secondo luogo, l'azione giudiziaria di accertamento del diritto dell'elettore di «votare conformemente alla Costituzione», essa (come pure già precisato) non smentisce la natura «politica» del sistema italiano. Il giudizio che ne consegue, infatti, non è idoneo ad incidere sulla validità del risultato elettorale - che resta rimessa alla decisione delle Assemblee di Camera e Senato ${ }^{24}$-, ma opera su un piano diverso (sebbene assai importante): quello della validità ed efficacia della legge elettorale. Si tratta dunque di un rimedio di natura giurisdizionale "complementare" alla «verifica dei poteri», e che arricchisce rispetto al passato gli strumenti a disposizione del cittadino per far valere l'eventuale lesione del suo diritto politico di voto (e i diritti connessi), anche in relazione alle elezioni del Parlamento ${ }^{25}$.

L'introduzione di questo strumento si deve, come già accennato, a due recenti sentenze della Corte Costituzionale: la n. 1/2014 e la n. 35/2017. O meglio: alla sentenza n. 1/2014, le cui conclusioni sono state confermate dalla Corte nella pronuncia del $2017^{26}$. La genesi della prima sentenza, in particolare, è abbastanza singolare. Alcuni cittadini comuni - mossi dall'intento di far rimuovere la legge elettorale allora vigente - propongono un ricorso al Tribunale civile di Milano in qualità di «cittadini elettori». Essi chiedono al Tribunale $(1)$ di dichiarare che il loro diritto di voto per eleggere le Camere «non può essere esercitato in modo libero e diretto»,

21 Per alcuni esempi di ciò (tratti soprattutto dall'esperienza delle prime legislature maggioritarie, successive alla "svolta" politicoistituzionale del 1993) v. L. Spadacini, Regole elettorali e integrità numerica delle Camere. La mancata assegnazione di alcuni seggi alla Camera nella XIV legislatura, Brescia, Promodis Italia editrice, 2003; M. Cerase, Sviluppi e contrasti in tema di verifica dei poteri, in Diritto pubblico, 2004, 662 ss.; G. Piccirilli, Contenzioso elettorale politico e verifica dei poteri: riflessioni sulla effettività delle tutele procedimentali, in Rassegna parlamentare, 2006, $788 \mathrm{ss.}$

22 Per quanto precede v., in particolare, L. Imarisio, Ineleggibilità e incompatibilità, cit., 292 ss.

23 Da ultimo, ordinanza n. 86/2020, che ha dichiarato inammissibile il ricorso per «conflitto di attribuzioni» proposto da un senatore del Movimento 5 Stelle contro l'assegnazione di un seggio ad un'altra deputata - da egli ritenuta illegittima - in seguito delle elezioni politiche del 2018.

24 Come ha affermato la Corte Costituzionale nella sentenza n. 1/2014, la dichiarazione di illegittimità della legge elettorale «produrrà i suoi effetti esclusivamente in occasione di una nuova consultazione elettorale ... Le elezioni che si sono svolte in applicazione anche delle norme elettorali dichiarate costituzionalmente illegittime costituiscono, in definitiva, e con ogni evidenza, un fatto concluso, posto che il processo di composizione delle Camere si compie con la proclamazione degli eletti» (p.to 7 del Considerato in diritto). 25 Oltre che a quelle degli organi elettivi locali e dei membri del Parlamento U.E., per le quali - come si è visto sopra - esistevano già prima diversi mezzi d'impugnazione giurisdizionale, e la connessa possibilità di sindacare la legittimità della disciplina legale in sede di giudizio «incidentale» di costituzionalità.

$26 \mathrm{Su}$ queste due decisioni, nell'amplissima bibliografia, si vedano in particolare M. D'Amico - S. Catalano (cur.), Prime riflessioni sulla "storica" sentenza 1 del 2014 in materia elettorale, Milano, Franco Angeli, 2014; G. Ferri (cur.), Corte Costituzionale e leggi elettorali delle Camere, Napoli, E.S.I., 2017; G. D'Amico, Azione di accertamento e accesso al giudizio di legittimità costituzionale, Napoli, Editoriale Scientifica, 2018; N. Zanon, Il controllo di costituzionalità sulle leggi elettorali politiche in Italia. Aspetti processuali (sentenze n. 1 del 2014 e $n .35$ del 2017), in www.cortecostituzionale.it. 
come previsto dalla Costituzione e dalla C.E.D.U. ${ }^{27}$, a causa del contenuto delle disposizioni della legge elettorale (legge n. 270/2005), e quindi (2) di «ripristinare tale diritto secondo modalità conformi alla legalità costituzionale». Al fine dell'accoglimento di tali domande $\mathrm{i}$ ricorrenti chiedono, inoltre, al giudice di sollevare davanti alla Corte Costituzionale (in via «incidentale») diverse questioni di costituzionalità delle norme della legge elettorale citata.

Questa azione è di ammissibilità molto dubbia in base al diritto processuale italiano, perché non mira a conseguire alcun "risultato utile" sul piano giuridico. Quand'anche, infatti, i ricorrenti fossero riusciti ad ottenere una sentenza di accertamento positivo del loro diritto di «votare conformemente alla Costituzione», essi non avrebbero potuto farla valere in concreto: né per far invalidare le elezioni svoltesi in passato (che rappresentavano un "fatto esaurito" non più contestabile, come ha confermato la sentenza n. 1/2014), né con riferimento alle elezioni future (in quanto il relativo risultato, come si è esposto sopra, nell'ordinamento italiano non può essere contestato davanti all'Autorità giudiziaria, ma solo tramite il procedimento interno al Parlamento della «verifica dei poteri») ${ }^{28}$. Inoltre, la richiesta dei ricorrenti di rimettere alla Corte Costituzionale le questioni di costituzionalità della legge elettorale sembra inammissibile anche per via dell'assenza di «pregiudizialità» tra il giudizio di legittimità costituzionale e l'oggetto del giudizio civile davanti al Tribunale. Nel caso in esame, infatti (come aveva evidenziato la dottrina), l'azione di accertamento «circa la lesione del diritto di voto dell'elettore coincide esattamente con l'azione di accertamento dell'illegittimità costituzionale della legge che disciplina quel voto ${ }^{29}$ : ciò contrasta con il necessario carattere «incidentale» (e "strumentale" alla pronuncia del giudice comune) del giudizio di costituzionalità nell'ordinamento italiano.

Nonostante queste apparenti preclusioni, il ricorso dei cittadini (dopo alterne vicende processuali) viene dichiarato ammissibile dalla Corte di Cassazione - giudice di ultimo grado - e le questioni di legittimità costituzionale vengono da essa sollevate davanti alla Corte Costituzionale. Quest'ultima, appunto con la sentenza n. 1/2014, conferma l'ammissibilità delle questioni, rilevando soprattutto l'esigenza di sottoporre al controllo di costituzionalità una legge fondamentale «per l'ordinamento costituzionale complessivamente considerato» come quella elettorale (evitando, dunque, il prodursi di una «una zona franca nel sistema di giustizia costituzionale ... in un ambito strettamente connesso con l'assetto democratico, in quanto

27 Segnatamente, art. 48 Cost. e Protocollo 1 CEDU.

28 Sui dubbi di ammissibilità dell'azione esercitata dai cittadini- elettori davanti al giudice ordinario v. G. D'Amico, Azione di accertamento, cit.; E. Grosso, Riformare la legge elettorale per via giudiziaria? Un'indebita richiesta di 'supplenza' alla Corte Costituzionale, di fronte all'ennesima disfatta della politica, in Rivista AIC, n. 4/2013; nonché, volendo, G. Sobrino, Il problema dell'ammissibilità, cit., 7 ss.

29 E. Grosso, op. cit., 2 
incide sul diritto fondamentale di voto» $\left.{ }^{30}\right)$. Nel merito, poi, la Corte Costituzionale accoglie le questioni e (per la prima volta nella sua storia) annulla le disposizioni della legge elettorale per l'elezione del Parlamento (in particolare quelle relative alla c.d. «formula elettorale») per contrasto con diverse norme della Costituzione.

Al di là della criticabilità, sotto certi profili, della scelta sull'ammissibilità delle questioni di legittimità costituzionale operata nella fattispecie dalla Corte $^{31}$, ciò che rileva ai fini della presente analisi è il "risultato" di tale orientamento. A partire dalla sentenza n. 1/2014, invero, qualsiasi cittadino è legittimato ad agire davanti a un giudice per accertare il suo diritto di «votare conformemente alla Costituzione» nelle elezioni politiche; e i giudici sono autorizzati, di conseguenza, a sollevare davanti alla Corte Costituzionale questioni di costituzionalità della legge elettorale del Parlamento, la quale può essere dichiarata invalida al pari di quelle per l'elezione degli organi locali e dei membri italiani del Parlamento U.E. (supra, § 3.1). Ciò infatti si è già verificato, poco dopo la pronuncia ricordata, con la sentenza n. 35/2017.

\section{4. - Il sistema messicano di giustizia elettorale: caratteri generali e principali}

\section{organi ed istituti}

Venendo ora al sistema di giustizia elettorale del Messico ${ }^{32}$, anch'esso, come quello italiano, muove da una caratterizzazione tradizionale quale modello di giustizia elettorale «politica» (competenza affidata, infatti, dalla Costituzione Federale del 1824 alle due Camere del Congresso). A differenza dell'Italia, però, nel corso della sua storia (che abbraccia un periodo più lungo: dal 1824, appunto ${ }^{33}$, fino ai giorni nostri) esso si è evoluto assai profondamente, sino a cambiare natura e - conseguentemente - classificazione tra i "tipi" di ordinamenti giuridico- processuali elettorali richiamati all'inizio di questo articolo (supra, § 2). Tenendo infatti conto del criterio di classificazione consistente nella natura dell'organo «incaricato di emettere la decisione finale sulle impugnazioni relative alla validità ed ai risultati delle elezioni ${ }^{34}$, il sistema di giustizia elettorale vigente oggi in Messico viene definito dalla dottrina più avvertita«pienamente giudiziario $\rangle^{35}$.

30 Così il punto 2 (ultimo capoverso) della motivazione della sentenza n. 1/2014. Utilizzando l'espressione «zona franca» la Corte Costituzionale ha fatto riferimento esplicito alla tradizionale dottrina costituzionalistica italiana, che - sia pure problematicamente aveva escluso la possibilità per la Corte stessa di sindacare la conformità alla Costituzione delle leggi elettorali della Camera e del Senato, in quanto leggi il cui rispetto è demandato dall'art. 66 Cost. al controllo degli organi parlamentari (in sede appunto di convalida degli eletti); e in ordine alle quali non si riteneva dunque possibile radicare un giudizio davanti a un giudice comune, nel quale eccepire questioni di costituzionalità (v. ad es. R. Bin, "Zone franche” e legittimazione della Corte, in Forum dei Quaderni costituzionali, 5 maggio 2014, e M. Manetti, L'accesso alla Corte costituzionale nei procedimenti elettorali, in A. Anzon - P. Caretti - S. Grassi (cur.), Prospettive di accesso alla giustizia costituzionale, Torino, Giappichelli, 2000, 136 ss.).

31 In particolare dal punto di vista della "forzatura" (per non dire violazione) delle regole del processo costituzionale - che si ripercuote sulla sua legittimazione nel sistema - e della possibile apertura all'accesso diretto al giudizio di legittimità costituzionale: sia consentito rinviare, al riguardo, a G. Sobrino, Il problema dell'ammissibilità, cit., 27 ss.

32 Per la cui descrizione che segue nel testo si farà riferimento principalmente ancora a J. Orozco Henriquez, Justicia electoral comparada, cit., 100 ss. (oltre che agli altri scritti di studiosi messicani indicati nella bibliografia in calce al presente articolo).

33 Il Messico è uno Stato indipendente dal 1821, ma la sua prima Costituzione è del 1824.

34 V. ancora supra, $\$ 2$.

35 J. Orozco Henriquez, Justicia electoral comparada, cit., 100. 
Tale qualificazione deriva dal fatto che da quasi venticinque anni (precisamente, da una riforma costituzionale del 1996), in base alla Costituzione ${ }^{36}$ ed alle fonti ad essa subordinate la «decisione finale» su tutti i ricorsi riguardanti lo svolgimento delle elezioni - compresa l'elezione del Presidente, la più importante in un sistema (ancora, a differenza dell'Italia) presidenziale - spetta ad un organo appartenente al potere giudiziario e avente natura, appunto, giurisdizionale: il Tribunal Electoral del Poder Judicial ${ }^{37}$. Esso (come vedremo meglio oltre) si configura come un organo «speciale» del potere giudiziario messicano, dotato di competenza esclusiva in materia elettorale; le sue decisioni - tra cui, appunto, la «dichiarazione di validità» del Presidente eletto - sono definitive e non impugnabili da alcun altro organo o potere dello Stato (o meglio: della Federazione, essendo il Messico uno Stato federale).

Per quanto le «decisioni finali» sul contenzioso elettorale spettino al Tribunal Electoral del Poder Judicial, nell'attuale ordinamento messicano riveste un ruolo fondamentale (accanto proprio al Tribunal Electoral) l'Instituto Nacional Electoral (I.N.E.). Si tratta di un organo istituito nel 2014, dalla trasformazione dell'Instituto Federal Electoral, che operava nel sistema dal 1990. Esso ha natura formalmente amministrativa, ma è dotato di rilevanti garanzie di autonomia ed indipendenza dal potere esecutivo (e, più in generale, dagli organi politici), similmente a quanto avviene in Italia, a partire dagli anni Novanta dello scorso secolo, per le cc.dd. «Autorità amministrative indipendenti». La sua competenza in materia elettorale può definirsi «generale»: come vedremo meglio in seguito, l'Instituto adotta tutti i provvedimenti che riguardano i partiti politici e la loro partecipazione (così come quella dei singoli candidati e dei cittadini- elettori) alle consultazioni elettorali; in particolare, esso si occupa di tutti gli aspetti (anche i più “minuti”) riguardanti l'organizzazione delle elezioni e la tutela dei diritti politico-elettorali dei cittadini. In tale contesto, l'I.N.E. è competente anche a decidere in prima istanza sugli eventuali ricorsi presentati dagli elettori; le sue decisioni sono impugnabili davanti al citato Tribunal Electoral (il quale - a differenza dell'I.N.E. - ha competenze esclusivamente giurisdizionali).

Il passaggio da un modello di giustizia elettorale «politica» al sistema attuale - come si è visto «pienamente giudiziario» - nell'ordinamento messicano non è stato, peraltro, immediato né diretto. A partire dal 1977 infatti, per effetto di una prima importante riforma, si è aperta una fase "intermedia" e di transizione dall'assetto originario all'altro, che la dottrina messicana oggi definisce (retrospettivamente) sistema di giustizia elettorale «misto». In questa fase, nel 1987, era stato istituito il Tribunal de lo Contencioso Electoral -primo Tribunale elettorale nella storia

36 Formalmente chiamata «Costituzione politica degli Stati Uniti Messicani», ed approvata originariamente nel 1917.

37 Questa soluzione - si noti - distingue l'ordinamento messicano dalla maggior parte dei sistemi di giustizia elettorale dei Paesi dell'America Latina: in essi l'organo “di vertice" del contenzioso elettorale, deputato ad adottare la «decisione finale» sulla validità ed i risultati delle elezioni, è un organo indipendente da tutti i poteri dello Stato (compreso il giudiziario) e riconducibile, perciò, alla categoria degli organi «di garanzia costituzionale» (come in Italia la Corte Costituzionale). 
del Messico -, trasformato nel 1990 nel Tribunal Federal Electoral, il "genitore" dell'attuale Tribunal Electoral del Poder Judicial. Sempre nel 1990, inoltre (come anticipato sopra), era stato creato l'Instituto Federal Electoral, diretto antecedente storico dell'I.N.E., il quale oggi rappresenta il secondo "asse portante" dell'ordinamento istituzionale elettorale. Tuttavia, fino a metà degli anni Novanta dello scorso secolo (riforma costituzionale del 1996, già citata), le decisioni del Tribunal Federal Electoral potevano essere "superate" o, comunque, modificate dalle Camere legislative, sia pure con un voto a maggioranza qualificata. Di qui, la connotazione non ancora propriamente «giudiziaria» - ma appunto, «mista»politico- giudiziaria, se non addirittura «politica» considerata la natura dell'organo deputato a decidere in ultima istanzadi tale modello ${ }^{38}$.

Sul piano della teoria generale, se (come abbiamo visto in precedenza a proposito dell'ordinamento italiano) l'adozione di un sistema di giustizia elettorale «politica» si fonda sul principio della separazione dei poteri inteso in senso «rigido», come nella concezione liberale ottocentesca di Stato, l'opzione a favore del modello «giurisdizionale» viene invece giustificata dalla dottrina - in Messico, ma non solo ${ }^{39}$ - con il principio di supremazia della Costituzione, e dei diritti politico- elettorali da essa sanciti, tipico della forma di Stato democraticocostituzionale contemporanea. Si osserva, al riguardo, che l'attribuzione ad un organo di natura giurisdizionale - indipendente, perciò, dal potere politico -, come il Tribunal Electoral del Poder Judicial, della competenza a decidere in ultima istanza sui ricorsi dei candidati e dei cittadini in materia elettorale serve a «garantire che tutti gli atti ed i provvedimenti elettorali si conformino inderogabilmente ai principi di costituzionalità e di legalità, e ad assicurare la protezione dei diritti fondamentali politico- elettorali dei cittadini di votare e di essere votati»: secondo il disposto dell'art. 41, paragrafo 2-VI, della Costituzione del Messico. «In questo senso - afferma la dottrina - il sistema di giustizia elettorale messicano ... ha come obiettivo garantire la vigenza dello Stato costituzionale democratico di diritto, il quale esige la celebrazione di elezioni libere, autentiche e periodiche, strettamente conformi alla Costituzione e alla legge». Inoltre (sotto un altro, ma correlato, profilo), si sottolinea che dal punto di vista del contenuto, «l'attività consistente nel giudicare e nel qualificare le elezioni ha concretamente natura giurisdizionale»; di conseguenza, «l'esercizio di tali attribuzioni deve essere rimesso ad un organo giurisdizionale, al fine di garantire l'autenticità, la regolarità e la validità delle elezioni» ${ }^{40}$.

Come si vede, si tratta di una prospettiva teorico- costituzionale del tutto diversa da quella

38 Per questa evoluzione storica e classificazione teorica, J. Orozco Henriquez, op. cit., 100 ss. e, più dettagliatamente, 133 ss. 39 Per quanto riguarda la dottrina italiana, in chiave evidentemente non di giustificazione del sistema vigente ma di proposta di riforma dello stesso nella direzione, appunto, della «giurisdizionalizzazione», v. per es. L. Imarisio, Ineleggibilità e incompatibilità, cit., 294 ss., in particolare 299.

40 Per tutte queste considerazioni v. sempre J. Orozco Henriquez, Justicia electoral comparada, cit., 61 e 142. 
sottesa al modello di giustizia elettorale «politica» (tipico, per quanto qui rileva, dell'Italia).

Passiamo, a questo punto, ad esaminare più nel dettaglio il sistema messicano di giustizia elettorale. Come preannunciato all'inizio di questo lavoro (supra, § 2), mi soffermerò, in particolare, sugli organi ed istituti (mezzi d'impugnazione ed altri strumenti di tutela) principali da esso previsti oggi, nel quadro di un ordinamento - è necessario avvertire - abbastanza complesso, di tipo federale (e quindi connotato dalla compresenza di regole adottate a livello centrale e di regole "locali" dei diversi Stati) e soggetto, infine, a cambiamenti e riforme abbastanza frequenti.

Iniziando dal Tribunal Electoral del Poder Judicial - organo "di vertice" di tale sistema, per quanto si è detto sopra -, esso è composto da una sezione «superiore» («Sala Superior») e da sei sezioni «regionali» («Salas Regionales») operanti a livello territoriale. Una di esse, peraltro, ha una competenza «specializzata» ma generale in materia di illeciti amministrativi relativi all'accesso dei partiti politici ai mezzi di comunicazione ed alla propaganda politica ( «Sala Regional Especializada»). La maggior parte delle decisioni complessivamente devolute al Tribunal Electoral (delle quali si dirà appresso) vengono adottate in un unico grado di giudizio, davanti alle sezioni «regionali»: solo alcuni dei mezzi d'impugnazione previsti nei confronti dei risultati elettorali, in particolare, possono essere proposti sia davanti a queste ultime che davanti alla «Sala Superior» in grado d'appello.

La «Sala Superior» è attualmente formata da sette magistrati, eletti dal Senato («Camara de Senadores») - organo quindi parlamentare - a maggioranza qualificata di due terzi, tra una "rosa” di candidati proposta dalla Suprema Corte di Giustizia («Suprema Corte de Justicia de la Nacion»): il che conferma la natura di organo «speciale» interno al potere giudiziario messicano del Tribunal Electoral. Tali magistrati restano in carica nove anni. I membri delle sezioni «regionali» sono invece tre; essi vengono eletti nello stesso modo e hanno un mandato di analoga durata. Sia la «Sala Superior» che le «Salas Regionales», peraltro, si configurano come organi «a rinnovo parziale e continuo» (come la Corte Costituzionale in Italia, in particolare) in quanto i loro giudici non vengono eletti (e quindi non scadono dal mandato) tutti insieme. Per ogni magistrato da sostituire, quindi, la Suprema Corte di Giustizia propone al Senato una terna di candidati.

Sul piano delle competenze - e quindi dei mezzi d'impugnazione che, in base alla Costituzione messicana ed alle fonti subordinate ${ }^{41}$, possono essere proposti dinanzi a tale organo -, il Tribunal Electoral è titolare del potere di decidere sui seguenti ricorsi: 1) ricorsi riguardanti le elezioni federali dei membri del Parlamento (deputati e senatori): c.d. «juicio de inconformidad» davanti alle sezioni «regionali»e «recurso de reconsideracion»

41 Specialmente la «Ley General del Sistema de Medios de Impugnación en Materia Electoral». 
davanti alla «Sala Superior»; 2) ricorsi relativi all'elezione del Presidente federale: «juicio de inconformidad» esclusivamente davanti alla «Sala Superior» (la quale, alla sua conclusione, emette in via definitiva la «dichiarazione di validità» dell'elezione presidenziale a cui si è accennato in precedenza); 3) ricorsi contro gli atti e le decisioni dell'Instituto Nacional Electoral (I.N.E.) diversi da quelli dei due punti precedenti (posto che l'I.N.E., come vedremo, dichiara i risultati di queste due elezioni): «recurso de apelacion» davanti alla «Sala Superior» o alle «Salas Regionales», a seconda delle materie; 4) ricorsi contro gli atti e le decisioni delle Autorità dei singoli Stati competenti in materia di organizzazione e di «qualificazione» («calificacion») delle consultazioni elettorali, e di risoluzione delle relative controversie: «juicio de revision constitucional electoral» davanti alla «Sala Superior» o alle «Salas Regionales», a seconda dei casi; 5) ricorsi contro gli atti e le decisioni delle Autorità federali e locali lesive dei diritti politico- elettorali dei cittadini (in particolare, diritto di elettorato attivo e passivo e di libera associazione politica): «juicio para la proteccion de los derechos politico-electorales del ciudadano» davanti alla «Sala Superior» o alle «Salas Regionales», a seconda dei casi; ๑) ricorsi del personale dello stesso Tribunal Electoral e dell'I.N.E. riguardanti il rispettivo rapporto di lavoro, esclusivamente davanti alla «Sala Superior»; 7) ricorsi contro le sanzioni irrogate dall'I.N.E. ai partiti, alle associazioni politiche ed ai singoli cittadini per la violazione di norme costituzionali o di legge.

A fronte di tale elenco assai nutrito (e rilevante soprattutto dal punto di vista del contenuto delle materie coinvolte), l'unica competenza di cui il Tribunal Electoral non è titolare in ambito elettorale consiste - è opportuno sottolinearlo in questa sede - nel controllo di costituzionalità «in astratto» delle leggi elettorali: ossia quello che in Italia, come abbiamo visto sopra, viene svolto dalla Corte Costituzionale con riferimento in particolare alla legge elettorale del Parlamento nazionale, in seguito alle sentenze n. 1/2014 e 35/2017. Infatti, nell'ordinamento messicano la c.d. «accion de incostitucionalidad» contro queste leggi deve essere esercitata - come in ogni altra materia - davanti alla Suprema Corte di Giustizia (da parte delle minoranze parlamentari, dei partiti politici registrati presso l'I.N.E. o a livello locale, del Procuratore Generale dello Stato e degli altri soggetti a ciò legittimati). Il Tribunal Electoral peraltro, osserva la dottrina, esercita un «controllo di costituzionalità nei casi concreti»: cioè valuta la conformità alla Costituzione degli specifici atti e provvedimenti in materia politicoelettorale indicati sopra, sottoposti alla sua cognizione ${ }^{42}$. Vi è dunque, sul punto, una divisione di competenze tra i due organi giudiziari.

In terzo luogo, oltre al Tribunal Electoral e (limitatamente al sindacato di costituzionalità «in astratto» delle leggi elettorali) alla Suprema Corte de Justicia de la Nacion, un organo 
fondamentale del sistema di giustizia elettorale del Messico è il già ricordato Instituto Nacional Electoral (I.N.E.), “erede” dell'Instituto Federal Electoral (I.F.E.), che ha operato dal 1990 al 2014. A differenza degli altri due organi, l'I.N.E. dal punto di vista formale è un'Autorità amministrativa: esso è titolare di una competenza "generalizzata" in materia politico- elettorale, che esercita sulla base, ed in attuazione, delle disposizioni di legge.

L'Instituto ha dunque una struttura articolata e gerarchica: essa comprende ${ }^{43}$ organi «centrali» e «locali» («organos delegacionales», costituiti dalle «Juntas Locales Ejecutivas» che operano nei 32 Enti federati del Messico e dalle «Juntas Distritales Ejecutivas» che operano nei 300 distretti elettorali), al di sotto dei quali vi sono uffici con competenze specifiche (essenzialmente, le «Direzioni esecutive» e le «Unità tecniche»). I principali organi «centrali» interni, che determinano e coordinano le attività dell'Instituto, sono il «Consiglio generale» («Consejo General») e la «Giunta generale esecutiva» («Juntas General Ejecutiva»). Il primo è composto da undici membri "pleno jure” («Consejeros Electorales») - compreso il Presidente -, eletti dalla Camera dei Deputati a maggioranza qualificata di due terzi tra una "rosa" di cinque candidati per ogni Consigliere proposta da un organo «tecnico» («Comitè Tecnico de Evaluacion»), designato a sua volta dalla Camera e da organi costituzionali autonomi. Tali membri durano in carica nove anni e non vengono eletti tutti insieme (essendo anche il «Consejo General» un organo «a rinnovo parziale e continuo»). Ad essi si aggiungono i membri rappresentanti del Parlamento («Consejeros del Poder Legislativo») - uno per ogni gruppo parlamentare di entrambe le Camere - ed i rappresentanti dei partiti politici («Representantes de los Partidos Políticos») - uno designato da ogni partito politico nazionale -, i quali possono intervenire alle sedute ma non hanno diritto di voto. La «Giunta generale esecutiva» invece (presieduta dal «Consejero Presidente») è formata dal «Segretario esecutivo» dell'Instituto e dai responsabili delle «Direzioni esecutive» e delle «Unità tecniche».

Alla luce della modalità di designazione dei membri "pleno jure" (cioè con diritto di voto) del «Consiglio generale» - rimessa ad uno dei due rami del Parlamento, con maggioranza qualificata, su proposta di un organo «tecnico» indipendente dal Governo -, si può convenire con la dottrina messicana nel qualificare l'I.N.E. come un «organo amministrativo elettorale autonomo» ${ }^{44}$. Si tratta, in effetti, di un Ente che ha attribuzioni «amministrative» (in una materia delicata come quella elettorale); ma con una posizione istituzionale «autonoma» ed indipendente - soprattutto dal potere esecutivo -, paragonabile (come già detto in precedenza) alle «Autorità amministrative indipendenti» dell'ordinamento italiano.

Venendo appunto alle competenze attribuite all'I.N.E. dalla legislazione messicana

43 V., al riguardo, anche l'apposita sezione del sito web istituzionale dell'Instituto: https://www.ine.mx/estructura-ine/.

44 J. Orozco Henriquez, Justicia electoral comparada, cit, in particolare 346 ss. (tabelle comparative dei sistemi di giustizia elettorale dell'America Latina). 
vigente (come modificata nel 2014, quando è stato creato l'Instituto), esse sono (principalmente) le seguenti ${ }^{45}:$ 1) l'emissione della «Credencial para votar», che in Messico - va sottolineato - non è "soltanto" la «tessera elettorale» necessaria per votare alle elezioni, ma assolve alla funzione generale di documento d'identità dei cittadini: di qui la grande importanza (e delicatezza) di questo compito dell'Instituto; 2) la registrazione dei partiti politici e delle associazioni politiche - nazionali e locali - ed il controllo delle loro attività, in particolare (ma non solo) ai fini della loro ammissione alle competizioni elettorali, sia nazionali che locali: in questo ambito l'I.N.E. è anche competente ad irrogare a tali soggetti politici le sanzioni per l'eventuale violazione delle norme che regolano la loro attività; 3) l'organizzazione delle consultazioni elettorali, a partire dalla fissazione preliminare degli «standards» organizzativi dei procedimenti elettorali (al fine di conformarli ai principi democratici) e dall'ammissione dei candidati (oltre che dei partiti di cui al punto precedente), fino alla dichiarazione dei risultati delle elezioni stesse; 4) l'informazione e l'assistenza e consulenza giuridica ai cittadini in materia elettorale, con riferimento, in particolare, ai ricorsi ed agli altri strumenti di tutela dei diritti politico- elettorale da essi esperibili; 5) come già accennato, la decisione in prima istanza su alcuni tipi di ricorsi presentati dagli elettori in relazione al procedimento ed ai risultati elettorali (le decisioni dell'I.N.E., in tal caso, sono impugnabili davanti al Tribunal Electoral); 6) lo sviluppo delle azioni relative alla protezione dei dati personali dei cittadini ed alla tutela del loro diritto di accesso alle informazioni pubbliche - con particolare riferimento all'attività dei partiti politici e degli organi di giustizia elettorale -, in chiave di realizzazione del principio democratico di trasparenza; 7) infine (in aggiunta a tali compiti propriamente «istituzionali»), la «comunicazione sociale» e la «promozione della cultura civica» e della tutela dei diritti all’interno della società messicana.

A dimostrazione dell'ampiezza e dell'importanza del ruolo dell'I.N.E. nell'ordinamento elettorale, ma più in generale nel sistema istituzionale e nel dibattito pubblico del Messico di oggi, si considerino questi dati: in sei anni dalla sua istituzione 1'I.N.E. ha organizzato ben 198 consultazioni elettorali, tra cui un'elezione del Presidente e tre del Parlamento federale (tutte le altre sono elezioni locali); queste elezioni hanno comportato, tanto a livello nazionale quanto a livello locale, un rilevante aumento dell'alternanza politica, il che - nel contesto messicano viene ritenuto indice dell' "impatto" positivo esercitato in concreto dall'attività di controllo e di informazione/sensibilizzazione in materia elettorale svolta dall'Instituto ${ }^{46}$.

In definitiva: se il Tribunal Electoral, come abbiamo visto, è la "cuspide" dell'attuale sistema di giustizia elettorale messicano - il suo "organo di vertice", competente a «decidere»

45 V., al riguardo, anche l'apposita sezione del sito web istituzionale dell'Instituto: https:/www.ine.mx/que-hace-el-ine/.

46 Questi dati e considerazioni sono tratti dall'intervento di Lorenzo Cordova Vianello (Presidente dell'I.N.E.) nel Seminario dal titolo «INE: de donde venimos y hacia donde vamos», svoltosi a Città del Messico, presso l'Instituto de Estudios para la Transicion Democratica (I.E.T.D.), il 25 gennaio 2020, al quale ho partecipato. 
in ultima istanza -, l'I.N.E. ne è il vero “baricentro". Ed è assai significativo, dall'angolo visuale dello studioso di diritto costituzionale italiano, che tale ruolo centrale in materia elettorale venga attribuito ad un organo del tutto indipendente dal Governo (e, più in generale, dalla politica). In Italia, infatti, la maggior parte delle competenze dell'I.N.E. - e nello specifico, quelle riguardanti il procedimento elettorale e l'organizzazione delle elezioni - è esercitata dal Ministero dell'Interno, organo proprio del Governo; né tali competenze, almeno per quanto concerne le elezioni del Parlamento nazionale, sono suscettibili di controllo da parte di organi giurisdizionali, come pure avviene in Messico.

E' opportuno ricordare ancora, ai fini della presente ricostruzione generale, che - essendo il Messico uno Stato federale - ai sensi degli artt. 116, paragrafo IV-d), e 122, paragrafo VII, della Costituzione ciascuno Stato, nell'esercizio della sua autonomia, stabilisce il proprio sistema «locale» di procedimenti elettorali e di mezzi d'impugnazione in materia, nonché gli organi - amministrativi e giurisdizionali - deputati ad esaminarli. Attualmente vi sono quindi, nell'ordinamento messicano, trentuno sistemi di giustizia elettorale (compreso quello di Città del Messico - una delle metropoli più popolose del mondo -, che costituisce un'entità federale autonoma). Peraltro, in seguito alla citata riforma costituzionale del 2014 che ha creato l'I.N.E., è in atto, secondo la dottrina, un processo di «centralizzazione della funzione elettorale»: esso si estrinseca, in particolare, nel fatto che i «Consejeros Electorales» ed il «Consejero Presidente» degli organismi elettorali «locali» sono designati (previo concorso pubblico) dal «Consiglio Generale» dell'I.N.E., e che i membri dei Tribunali elettorali «locali» sono eletti dal Senato federale (con una maggioranza qualificata di due terzi). Inoltre l'I.N.E. ha il potere di «avocare» (a livello «centrale» quindi) le funzioni degli organi «locali» in materia elettorale o di sostituirsi ad essi nell'adozione di specifiche decisioni ${ }^{47}$, con un voto a maggioranza qualificata di otto undicesimi del «Consiglio Generale». In ogni caso, i provvedimenti delle Autorità elettorali «locali» (come si è già accennato) sono tutti impugnabili davanti al Tribunal Electoral del Poder Judicial, a seconda dei casi dinanzi alla «Sala Superior $\rangle^{48}$ o alla «Sala Regional» competente per territorio ${ }^{49}$.

Ricapitolando dunque (e per concludere l'esame del sistema messicano di giustizia elettorale) i principali mezzi d'impugnazione in materia elettorale esperibili oggi dai cittadini, dai candidati o dagli altri soggetti legittimati, essi sono ${ }^{50}$ : 1) il «recurso de revision», nei confronti degli atti o delle «omissioni» dell'I.N.E., proponibile davanti all'organo gerarchico superiore dello stesso I.N.E.;2) il «recurso de apelacion», nei confronti principalmente di alcuni

\footnotetext{
47 Per esempio, sulla capacità elettorale dei cittadini, sul "disegno" dei collegi elettorali, sulle liste degli elettori e dei candidati.

48 In particolare, risultati delle elezioni dei Governatori statali.

49 Per quanto precede v. J. Orozco Henriquez, Justicia electoral comparada, cit., 143 ss.

$50 \mathrm{~V}$. anche, al riguardo, la tabella comparativa dei mezzi d'impugnazione in materia elettorale degli ordinamenti dell'America Latina pubblicata in J. Orozco Henriquez, Justicia electoral comparada, cit., 359 ss. (il Messico è a pagina 371).
} 
provvedimenti dell'I.N.E., proponibile davanti alla «Sala Superior» o alle «Salas Regionales» del Tribunal Electoral del Poder Judicial; 3) il «procedimento especial sancionador», nei confronti delle sanzioni in materia politico- elettorale irrogate dall'I.N.E. o da altre Autorità competenti, proponibile davanti alla «Sala Regional Especializada» del Tribunal Electoral (ricordata in precedenza); 4) il «juicio de inconformidad», nei confronti dei risultati delle elezioni del Presidente e dei membri del Parlamento (deputati e senatori), esperibile, rispettivamente, davanti alla «Sala Superior» o alle «Salas Regionales» territorialmente competenti del Tribunal Electoral; 5) il «recurso de reconsideracion», nei confronti delle decisioni sul «juicio de inconformidad» delle elezioni dei membri del Parlamento, proponibile davanti alla «Sala Superior» del Tribunal Electoral; 6) il «juicio para la proteccion de los derechos politicoelectorales del ciudadano», nei confronti degli atti di approvazione delle liste elettorali e degli atti lesivi dei diritti politico- elettorali dei cittadini (in particolare, diritto di elettorato attivo e passivo e di libera associazione politica), esperibile davanti alla «Sala Superior» o alle «Salas Regionales» del Tribunal Electoral, a seconda dei casi; 7) il «juicio de revision constitucional electoral», nei confronti degli atti e delle decisioni delle Autorità elettorali «locali», esperibile davanti alla «Sala Superior» o alle «Salas Regionales» del Tribunal Electoral, a seconda dei casi; 8) la «revision», nei confronti delle decisioni dei procedimenti sanzionatori contro i partiti politici e delle «misure cautelari» relative alla comunicazione radiotelevisiva elettorale, esperibile davanti alla «Sala Superior» del Tribunal Electoral.

\section{5. - Osservazioni finali}

Volendo trarre dall'analisi svolta alcune sintetiche (e, per quanto detto all'inizio di questo articolo, "provvisorie") conclusioni di carattere generale, può osservarsi che i due sistemi di giustizia elettorale esaminati - quello italiano e quello messicano - rappresentano "modelli" diversi, ispirati a logiche e principi in gran parte differenti.

Il primo infatti - quello italiano -, come si è visto, è un sistema connotato ancora oggi prevalentemente da una giustizia (o da un «contenzioso») elettorale «politica»: le impugnazioni riguardanti l'elezione dei membri del Parlamento sono trattate e decise, in via insindacabile, dalle stesse Camere, senza possibilità di intervento di organi diversi (di natura giudiziaria o, come avviene in altri ordinamenti costituzionali, per esempio dell'America Latina, di garanzia costituzionale). La logica alla base di questa impostazione risiede nella volontà di sottrarre ad organi esterni, appunto, al potere politico le decisioni in grado di incidere sulla composizione (e sul conseguente funzionamento) del Parlamento, secondo una concezione «rigida» e tradizionale della separazione dei poteri di matrice liberale ottocentesca.

L'attuale ordinamento messicano invece, come si è visto nell'ultimo paragrafo, esprime 
un "modello" di giustizia elettorale chiaramente (e «pienamente» $\left.{ }^{51}\right)$ «giudiziario». In esso infatti - al contrario che in Italia - la «decisione finale» sui ricorsi riguardanti lo svolgimento delle elezioni politiche, sia a livello «centrale» (membri delle due Camere e Presidente, nell'ambito di una forma di governo presidenziale) che a livello «locale», è affidata ad un organo «speciale» appartenente al potere giudiziario: il Tribunal Electoral del Poder Judicial. Questa scelta, sul piano della teoria generale, si giustifica in primo luogo con il principio di supremazia della Costituzione (e dei relativi diritti politico- elettorali riconosciuti ai cittadini) tipico dello Stato democratico- costituzionale contemporaneo, che esige che tutti gli atti del potere politico - a partire dalla formazione/costituzione degli organi rappresentativi - siano sottoposti al controllo di legittimità (e, in particolare, di costituzionalità) di organi indipendenti dalla politica stessa. In secondo luogo, essa si giustifica con il rilievo della natura sostanzialmente giurisdizionale dell'«attività consistente nel giudicare e nel qualificare le elezioni»: «l'esercizio di tali attribuzioni - si osserva al riguardo - deve essere rimesso ad un organo giurisdizionale, al fine di garantire l'autenticità, la regolarità e la validità delle elezioni»» ${ }^{52}$.

Queste differenze tra i due sistemi, e la loro rispettiva attuale connotazione, può spiegarsi, tra gli altri fattori, con la diversità dei contesti politico- istituzionali dei due Paesi in esame (posto che tutti gli ordinamenti positivi sono, in qualche misura, il "prodotto" di uno specifico contesto politico- istituzionale, oltre che sociale, culturale, ecc.).

Da questo punto di vista, va osservato che l'Italia - pur con le sue note peculiarità e debolezze storiche - si colloca in una tradizione europea di matrice liberale. Anche la formazione del suo sistema di «contenzioso»/giustizia elettorale è stata influenzata, dunque, dalla concezione liberale della «sovranità del Parlamento»: come dimostra il fatto che l'istituto della «verifica dei poteri» è stato introdotto, durante il periodo statutario (con una soluzione poi trasposta nell'art. 66 della Costituzione del 1948), mutuandolo dall'ordinamento inglese ${ }^{53}$.

Dall'altro lato, il Messico, per ragioni storiche ed anche di collocazione geografica, non ha subito un'analoga influenza (almeno, non in termini così netti); esso piuttosto - soprattutto in una fase recente della sua storia, collocabile negli ultimi decenni del Novecento - ha dovuto affrontare il problema del consolidamento della sua democrazia, minacciata da vari fenomeni: uno su tutti la corruzione politica (con il connesso "carico" di violenza nel Paese). In questo contesto, come osserva la dottrina messicana, la costruzione di un sistema di giustizia elettorale completo, per certi versi "sofisticato" - si pensi al numero ed alla tipologia dei mezzi d'impugnazione precedentemente ricordati - e, soprattutto, affidato ad organi indipendenti dalla politica (Tribunal Electoral, ma in particolare, per la sua centralità nel sistema, l'I.N.E.) è

51 J. Orozco Henriquez, Justicia electoral comparada, cit., 100.

52 Ibidem, 61 e 142.

53 L. Imarisio, Ineleggibilità e incompatibilità, cit., 295. 
risultata strumentale alla protezione ed al rafforzamento della democrazia ed all'incremento di tutela dei diritti - in primo luogo politici - dei cittadini messicani ${ }^{54}$.

Dal punto di vista (invero decisivo) della "resa" dei due sistemi di giustizia elettorale qui considerati - ossia dell'effettività della tutela dei diritti politico- elettorali dei cittadini da essi apprestata ${ }^{55}$, è interessante osservare la differenza di valutazioni che ne viene data "dall'interno": cioè, rispettivamente, dalla dottrina italiana e messicana.

Quanto all'Italia, la prima - pur riconoscendo che «nei primi decenni dell'esperienza repubblicana il sistema delineato dalla Costituzione ... non ha ... dato luogo a frequenti o eclatanti episodi di distorsioni politiche nell'impiego dello strumento della verifica dei poteri» - oggi manifesta una diffusa insoddisfazione per il "modello" «politico». Essa sottolinea al riguardo che, soprattutto in un contesto di parlamentarismo (più o meno marcatamente) maggioritario, come quello instauratosi in Italia negli ultimi decenni, è necessario «prevenire ... i rischi connessi con l'assenza, nel modello vigente, di adeguate garanzie in ordine alla verifica delle elezioni per la posizione dei componenti dell'opposizione parlamentare. L'adozione di un sistema implicante, anche in forma successiva ed eventuale rispetto al giudizio interno, l'intervento di un controllo di tipo giurisdizionale - si conclude di conseguenza - appare ... maggiormente conforme agli attuali assetti ed equilibri politico- istituzionali dell'ordinamento» italiano $^{56}$.

Invece, per quanto riguarda il sistema di giustizia elettorale del Messico, la dottrina messicana esprime - in modo concorde - un giudizio ampiamente positivo. In proposito è stato affermato, emblematicamente, che «il passaggio da un sistema di giustizia elettorale affidato principalmente ad un organo legislativo o politico ... ad un altro di natura giurisdizionale (in poco più di trent'anni, a partire dall'istituzione del primo Tribunal Electoral) ha comportato un cambiamento nella cultura giuridica e politica che si è consolidato rapidamente ..., permettendo la formazione di un autentico sistema integrale di giustizia elettorale in grado di garantire lo svolgimento di elezioni (tanto federali quanto locali) libere, autentiche e periodiche, strettamente vincolate alla Costituzione e alla legge» ${ }^{57}$. Per questo motivo vengono guardati con sospetto, se non apertamente criticati, i tentativi della politica (ricorrenti soprattutto nell'ultimo periodo) di intervenire sull'ordinamento vigente, per esempio modificando il sistema di nomina dei Consiglieri e del Presidente dell'I.N.E., eliminando gli organi elettorali «locali» oppure

54 Come ha affermato Lorenzo Cordova Vianello (Presidente dell'I.N.E.) nel già ricordato Seminario «INE: de donde venimos $y$ hacia donde vamos» del 25 gennaio 2020, «le riforme istituzionali e la domanda di democrazia da parte dei cittadini hanno condotto gradualmente ad un sistema politico più inclusivo, aperto e democratico»; soprattutto le riforme dopo il 1996 (cfr. supra, § 4) hanno inteso «rafforzare l'equità nella competizione elettorale e consolidare gli strumenti, i processi e le pratiche» della giustizia elettorale. 55 Aspetto che, beninteso, richiederebbe un'analisi ben più approfondita e circostanziata di quella che ho svolto fino a questo momento. 56 Per quanto precede, L. Imarisio, Ineleggibilità e incompatibilità, cit., 296 e 299 (corsivi miei).

57 Così J. Orozco Henriquez, Justicia electoral comparada, cit., 149 (corsivi miei). Per alcuni esempi di recenti decisioni giudiziarie che, per la dottrina, hanno contribuito a questo risultato v. F.A. Fuentes Barrera - P. Salazar Ugarte (cur.), Memoria del I Conversatorio de Sentencias Relevantes en Materia Electoral, Mexico, U.N.A.M.- T.E.P.J.F., 2019. 
attraendo dall'I.N.E. stesso al Governo la competenza relativa al rilascio del «padròn electoral», che, come si è visto, funge da vero e proprio documento d'identità dei cittadini ${ }^{58}$.

In effetti, anche alla luce di tali concordi valutazioni degli studiosi di diritto elettorale italiani e messicani, la soluzione «giurisdizionale» circa la conformazione del sistema di giustizia elettorale (intendendosi per tale, come detto sopra, quella che affida ad organi di natura giurisdizionale almeno la «decisione ultima» sui ricorsi contro gli atti ed i risultati delle elezioni) sembra preferibile: essa pare più coerente con i principi dell'odierno Stato democratico costituzionale. In questa prospettiva, dovrebbe essere l'ordinamento italiano ad "avvicinarsi" a quello messicano; non viceversa. L'istituzione di un organo giurisdizionale ad hoc, «speciale», esclusivamente competente ad esaminare le impugnazioni contro le delibere del Parlamento riguardanti la «verifica dei poteri» e la conseguente assegnazione dei seggi, è senza dubbio un'utile suggestione (forse più che l'affidamento dei compiti amministrativi in materia di elezioni ad un organo autonomo dal potere politico come l'I.N.E., del quale in Italia si avverte meno l'esigenza). Peraltro, considerato (anche qui) lo specifico contesto istituzionale italiano e i delicati rapporti tra potere giudiziario e politica che lo caratterizzano, si potrebbe riflettere sull'opportunità di attribuire tale competenza, più che appunto ad un organo interno a tale potere, ad un soggetto autonomo da entrambi e con competenze tipiche di garanzia costituzionale, come la Corte Costituzionale (nella sua composizione ordinaria o forse meglio, in alternativa, attraverso una sua sezione "distaccata").

\section{Bibliografia}

C.E. Arenas Batiz - J. Orozco Henriquez, Derecho electoral, in Enciclopedia Juridica Mexicana, Mexico, Porrùa- U.N.A.M., 2002, vol. IX

F. Bailo, Capacità elettorale e Costituzione, Napoli, Jovene, 2015

V. Bazan, Control de las omisiones incostitucionales e inconvencionales. Recorrido por el derecho y la jurisprudencia americana y europea, Mexico, T.E.P.J.F., 2017

R. Bin, "Zone franche” e legittimazione della Corte, in Forum dei Quaderni costituzionali, 5 maggio 2014

R. Bin, La Corte Costituzionale può introdurre con una sentenza il ricorso diretto di costituzionalità delle leggi?, in laCostituzione.info, 13 gennaio 2017

E. Catelani - E. Donati - M.C. Grisolia (cur.), La giustizia elettorale, Napoli, Editoriale Scientifica, 2013

M. Cerase, Sviluppi e contrasti in tema di verifica dei poteri, in Diritto pubblico, 2004,

58 Queste proposte sono state criticate, per esempio, nel ricordato Seminario «INE: de donde venimos y hacia donde vamos» del 25 gennaio 2020, in quanto comporterebbero un arretramento del modello di giustizia elettorale messicano, con particolare riferimento al principio di autonomia degli organi elettorali. 
662 ss.

H.A. Concha Cantù - S. Lopez Noriega, La (in)justicia electoral a examen, Mexico, U.N.A.M., Instituto de Investigaciones Juridicas, C.I.D.E., 2016

L. Cordova Vianello - P. Salazar Ugarte, Democracia sin garantes. Las autoridades vs. la reforma electoral, Mexico, U.N.A.M., 2009

L. Cordova Vianello - P. Salazar Ugarte (cur.), Estudios sobre la reforma electoral 2007. Hacia un nuevo modelo, Mexico, T.E.P.J.F., 2008

G. D’Amico, Azione di accertamento e accesso al giudizio di legittimità costituzionale, Napoli, Editoriale Scientifica, 2018

M. D’Amico - S. Catalano (cur.), Prime riflessioni sulla "storica” sentenza 1 del 2014 in materia elettorale, Milano, Franco Angeli, 2014

L. Elia, Elezioni politiche (contenzioso), in Enciclopedia del diritto, Milano, Giuffrè, 1965, vol. XIV, 748 ss.

G. Ferri (cur.), Corte Costituzionale e leggi elettorali delle Camere, Napoli, E.S.I., 2017

H. Fix- Zamudio, Justicia constitucional y judicializacion de la politica, in J. Orozco Henriquez (cur.), Sistemas de justicia electoral. Evaluacion y perspectivas, Mexico, I.F.E.P.N.U.D.- U.N.A.M.- I.F.E.S.- I.D.E.A.- T.E.P.J.F., 2001

F.A. Fuentes Barrera - P. Salazar Ugarte (cur.), Memoria del I Conversatorio de Sentencias Relevantes en Materia Electoral, Mexico, U.N.A.M.- T.E.P.J.F., 2019

E. Grosso, Riformare la legge elettorale per via giudiziaria? Un'indebita richiesta di 'supplenza' alla Corte Costituzionale, di fronte all'ennesima disfatta della politica, in Rivista AIC, n. 4/2013

L. Imarisio, Ineleggibilità e incompatibilità politico- istituzionali, Napoli, Jovene, 2008

L. Lara Sàenz, Estudio de derecho comparado sobre lo contencioso y la jurisprudencia electoral en Italia, in Aa.Vv., El contencioso y la jurisprudencia electorales en el derecho comparado. Un estudio sobre 21 paises de América y Europa, Mexico, T.E.P.J.F., 2006

E. Lehner, Le garanzie dei diritti elettorali, Roma, Aracne, 2012

E. Lehner, Il diritto di voto dopo la conquista della "zona franca", in Giurisprudenza costituzionale, n. 1/2014, 54 ss.

M. Luciani, Bis in idem: la nuova sentenza della Corte costituzionale sulla legge elettorale politica, in Rivista AIC, n. 1/2017

M. Manetti, L'accesso alla Corte costituzionale nei procedimenti elettorali, in A. Anzon - P. Caretti - S. Grassi (cur.), Prospettive di accesso alla giustizia costituzionale, Torino, Giappichelli, 2000, 136 ss.

D. Nohlen - L. Valdés - D. Zovatto (cur.), Derecho electoral latinoamericano. Un 
enfoque comparativo, Mexico, F.C.E. - U.N.A.M.- I.N.E.- I.I., 2019

J. Orozco Henriquez, El sistema interamericano y la proteccion de los derechos politicos, in Id., Proteccion a los derechos humanos. Perspectivas nacionales e interamericanas, Mexico, Porrùa- U.N.A.M.- El Colegio Nacional- Instituto Mexicano de Derecho Procesal Constitucional, 2018

J. Orozco Henriquez, Justicia electoral comparada de América Latina, Mexico, U.N.A.M., Instituto de Investigaciones Juridicas, 2019

G. Piccirilli, Contenzioso elettorale politico e verifica dei poteri: riflessioni sulla effettività delle tutele procedimentali, in Rassegna parlamentare, 2006, 788 ss.

G. Sobrino, Il problema dell'ammissibilità delle questioni di legittimità costituzionale della legge elettorale alla luce delle sentenze n. 1/2014 e n. 35/2017 e le sue possibili ricadute: dalla (non più tollerabile) "zona franca" alla (auspicabile) "zona a statuto speciale" della giustizia costituzionale?, in federalismi.it, n. 15/2017

L. Spadacini, Regole elettorali e integrità numerica delle Camere. La mancata assegnazione di alcuni seggi alla Camera nella XIV legislatura, Brescia, Promodis Italia editrice, 2003

A. Vuolo, La legge elettorale. Decisione politica, controllo, produzione giurisprudenziale, Napoli, Editoriale Scientifica, 2017

N. Zanon, Il controllo di costituzionalità sulle leggi elettorali politiche in Italia. Aspetti processuali (sentenze n. 1 del 2014 e n. 35 del 2017), in www.cortecostituzionale.it 


\section{SOBRE O AUTOR:}

Giorgio Sobrino é doutor em Direito pela Università degli Studi di Torino. É pesquisador de Direito Constucional junto à Università degli Studi di Torino e advogado. É autor de Il Ministro della Giustizia ed i Poteri dello Stato. Vicende e prospettive di una collocazione problematica (ESI, 2015), dentre outros textos.

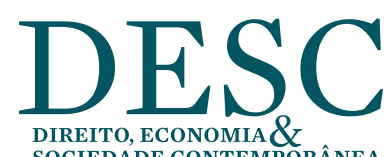

SOCIEDADE CONTEMPORÂNEA 